\title{
A Possible Role for the FKBP5 Gene in Schizophrenia
}

\author{
Qing Gao ${ }^{1}$, Zhe Li ${ }^{1}$, Paul Jones ${ }^{2}$, Guyu Zhang ${ }^{3^{*}}$ \\ ${ }^{1}$ The Mental Health Center and the Psychiatric Laboratory, West China \\ Hospital, Sichuan University, Chengdu, Sichuan, 610041, China; \\ ${ }^{2}$ Department of Biology Products, American Informatics Consultant LLC, \\ Rockville, MD, 20852, USA; \\ ${ }^{3}$ Spark Energy LLC, Houston, TX 77253, USA.
}

First Author: Qing Gao, The Mental Health Center and the Psychiatric Laboratory, West China Hospital, Sichuan University, Chengdu, Sichuan, 610041, China.

Joint First Author: Zhe Li, The Mental Health Center and the Psychiatric Laboratory, West China Hospital, Sichuan University, Chengdu, Sichuan, 610041, China.

*Corresponding Author: Guyu Zhang, Spark Energy LLC, Houston, TX, 77253, USA; gzhang@sparkenergy.com. Tel:225-304-1636.

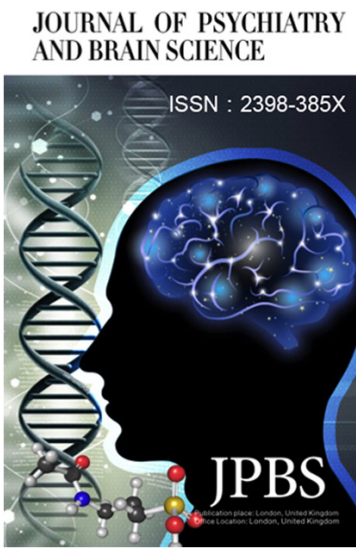

http://jpbs.qingres.com

\section{GOPEN ACCESS}

DOI: 10.20900/jpbs.20160020

Received: September 15, 2016

Accepted: November 12, 2016

Published: December 25, 2016

Copyright: $\odot 2016$ Cain et al. This is an open access article distributed under the terms of the Creative Commons Attribution License, which permits unrestricted use, distribution, and reproduction in any medium, provided the original author and source are credited.

\section{ABSTRACT}

Background: Recently, several studies reported possible association between gene FKBP5 and schizophrenia (SCZ). Their results suggested that FKBP5 may link to stress and cognition in case of SCZ, which warranted further study in the field.

Method: In this study, we performed a systematical literature review, focusing on FKBP5--brain region association studies and brain region-SCZ relations, with the purpose of identifying potential FKBP5 $\rightarrow$ brain $\rightarrow$ SCZ pathogenic pathways.

Results: We identified over 1,500 references supporting the relation of four common brain regions that related to both FKBP5 and SCZ. These brain regions include hypothalamus, hippocampus, prefrontal cortex, amygdaloid. Analysis showed that FKBP5 affects the structure and function of these four brain regions with different mechanisms, which may in turn influence the pathogenesis of SCZ.

Conclusion: Our results support the hypothesis that FKBP5 present linkage to the development of SCZ, which help to gain better understanding of the underlying FKBP $5 \rightarrow \mathrm{SCZ}$ association mechanism.

Keywords: FKBP5; Schizophrenia; hypothalamus; hippocampus; prefrontal cortex; amygdaloid

\section{INTRODUCTION}


Schizophrenia (SCZ) is one of the most chronically disabling psychiatric illnesses with a global median lifetime morbid risk of 7.2/1000 persons ${ }^{[1]}$. Early manifestations of the illness often appear in mid to late adolescence, with clinical onset typically following 2-5 years later. In clinical settings the disorder is identified through the presence of impairments across several clusters of symptoms, namely positive, negative, and cognitive symptoms, disorganization, altered mood and motor symptoms. Although etiology of SCZ remains unclear, recent neuroimaging studies provide compelling evidence of abnormalities of cerebral structure and function in patients with $\mathrm{SCZ}^{[2-4]}$. For instance, reductions in the volume of the hippocampus and the amygdala are consistent findings in imaging studies of $\mathrm{SCZ}{ }^{[5]}$, while enlarged hypothalamic volumes in SCZ have also been identified ${ }^{[6]}$. Moreover, SCZ patients tend to present functional dysconnectivity within/between several brain regions, including prefrontal cortex, hypothalamus, hippocampus, and amygdaloid ${ }^{[6-10]}$. These studies suggest that the genes linked to functional or structural changes of these SCZ-related brain regions are worthy of further study.

The protein encoded by gene FKBP5 is a member of the immunophilin protein family, playing roles in immunoregulation and basic cellular processes involving protein folding and trafficking. This gene is a key molecule in the stress response and the pathophysiology of psychiatric symptoms. Studies showed that FKBP5 plays a role as a glucocorticoid receptor (GR) regulator, influencing the GR binding and translocation by binding and initiating intracellular feedback on the GR system ${ }^{[11]}$. FKBP5 inhibits the function of GRs, which regulate adrenocortical secretion of glucocorticoids during stress-induced Hypothalamic pituitary adrenal (HPA) axis activity. The activation of the HPA axis is controlled through a negative feedback mechanism -by the activation of GRs at different levels of FKBP5 ${ }^{[12,13]}$. Under the pathological conditions, the function of the HPA axis is disrupted, and this may eventually lead to structural and functional changes in brain regions ${ }^{[14,15]}$.

The HPA-axis-regulation function of FKBP5 has been confirmed later by other studies, which were observed within many brain regions ${ }^{[12]}$. It has been demonstrated that HPA axis is directly involved in inducing mood behavior after chronic social defeat stress ${ }^{[16]}$. These findings explain the results from some other genetic studies that linked FKBP5 with posttraumatic stress disorder, depression an $d$ anxiety ${ }^{[17-19]}$. For instance, Yehuda et al. found that the single nucleotide polymorphisms (SNPs) in FKBP5 interacts with childhood trauma and could be used to predict severity of adult posttraumatic stress disorder (PTSD) ${ }^{[18]}$.

On the other hand, HPA axis dysregulation is suggested as one of the possible mechanisms related to the vulnerability-stress model of $\mathrm{SCZ}{ }^{[20]}$, posing a functional linkage between FKBP5 and SCZ. As a matter of fact, several recently studies reported possible association between the genetic changes of FKBP5 with the stress and cognition in case of SCZ ${ }^{[20-22]}$. However, further studies are need on exploring the functional mechanisms of FKBP5 that affect the pathogenic development of SCZ.

In this study, we conducted a systematically review on the association between FKBP5 and the brain regions, abnormalities of which are related to SCZ. Our study identified four potential FKBP5 $\rightarrow$ brain regions $\rightarrow$ SCZ pathogenic pathways through which FKBP5 influence the symptoms and development of SCZ.

\section{MATERIALS AND METHODS}

The literature reference selection criteria is described as follows. We searched the whole PubMed abstracts and approximately 4M+ Elsevier full journal papers, looking for two groups of papers: first, papers describing relationships between FKBP5 and its related brain regions; second, papers reporting SCZ related brain regions. There are 20 brain regions demonstrated functionally relation with FKBP5, and 134 brain regions related to schizophrenia. Then, we further selected the ones from these references that contain brain regions associated with both FKBP5 and SCZ. We identified four brain regions that demonstrate upstream regulations with FKBP5 (FKBP5 $\rightarrow$ Brain Regions), meanwhile downstream regulation with $\mathrm{SCZ}$ (Brain Region $\rightarrow$ SCZ). After manually inspection, we finally picked up 1,561 references and present them in Supplementary Data, including the titles and related sentences where these FKBP5-gene or SCZgene were identified. These references include 129 papers reporting relationships between FKBP5 and 4 brain regions (i.g., hypothalamus, Hippocampus, prefrontal cortex, amygdaloid). Also included are three references claim direct linkage between SCZ and FKBP5. The rest 1,428 references are these supported relationships between $\mathrm{SCZ}$ and the four brain regions mentioned above. Our study identified four gene $\rightarrow$ brain $\rightarrow$ disease pathogenic pathways, as shown in Fig 1 The detailed description of the construction of these pathways are presented in the following sections. 

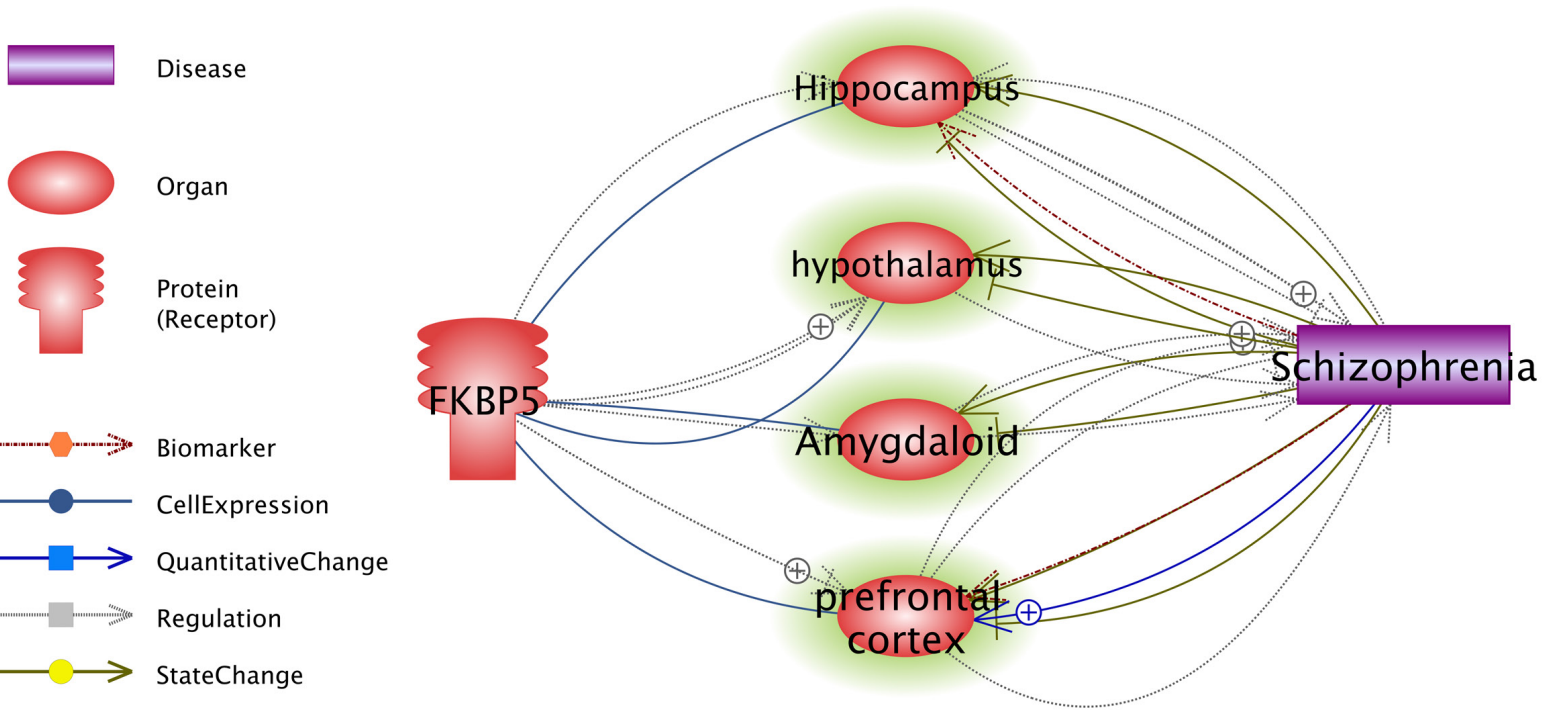

Fig. 1 Possible FKBP5 $\rightarrow$ brain $\rightarrow$ SCZ pathogenic pathways identified through systematic literature review. The four brain regions were highlighted in green.

\subsection{FKBP $5 \rightarrow$ brain region pathways regulating $S C Z$}

In this section, we present the detailed discussion on four potential FKBP5 $\rightarrow$ brain $\rightarrow$ SCZ pathogenic pathways. These pathways may contribute to a better understanding of the mechanisms of how FKBP5 could play roles in the pathogenic development of SCZ. The four brain regions involved in the discussion are hypothalamus, hippocampus, prefrontal cortex, amygdaloid. All these brain regions demonstrated strong association with SCZ, both functionally and structurally.

\subsection{FKBP5- Hippocampus-SCZ}

Hippocampus is a major component of the brains regions of humans and other vertebrates, which is densely populated with glucocorticoid receptors (GRs) ${ }^{[23]}$. Studies showed that persistent dysregulation of GR subcellular distribution is predicted to damage the hippocampal formation ${ }^{[24]}$. Highly expressed in the hippocampus, FKBP5 has been shown to regulate the steroid receptor function, including progesterone, androgen, and GRs ${ }^{[11]}$. By altering GR sensitivities, FKBP5 influences the function and morphology of hippocampal ${ }^{[25]}$. It has been identified that FKBP5 expression in hippocampus is also linked to reduced short term memory ${ }^{[26]}$, altered cortisol negative feedback ${ }^{[27]}$ and chronic mild stress (CMS) ${ }^{[28]}$.
On the other hand, studies indicated that functional abnormalities in the hippocampus, as well as hippocampal volume reductions may be a vulnerability marker for SCZ ${ }^{[10,29-32]}$. Therefore, structural deficits or dysfunction in the hippocampus resulted from the alteration of FKBP5 expression and GRs could build up a possible FKBP5 $\rightarrow$ Hippocampus $\rightarrow$ SCZ pathway linking FKBP5 to SCZ.

\subsection{FKBP5-hypothalamus-SCZ pathway}

Hypothalamus is a portion of the brain that contains a number of small nuclei with a variety of functions. One of the most important functions is to link the nervous system to the endocrine system via the pituitary gland (hypophysis). FKBP5 has been suggested as one of the key modulators of hypothalamic pituitary adrenal axis (HPA axis) ${ }^{[33-}$ ${ }^{35]}$, which controls reactions to stress and regulates many body processes, including digestion, the immune system, mood, emotions, sexuality, energy storage and expenditure. The activation of the HPA axis is controlled through a negative feedback mechanism, by the activation of GRs at different levels of FKBP5 ${ }^{[12,13]}$. For example, Albu et al. found that FKBP5 plays an important regulatory role in the HPA axis necessary for stress adaptation and recovery ${ }^{[33]}$. Fujii et al. also suggested that FKBP5 variant (rs1360780) regulates HPA axis 
reactivity and expression levels of $\mathrm{GR}^{[34]}$, which regulate adrenocortical secretion of glucocorticoids during stress-induced HPA axis activity. Other studies shown that chronic treatment with corticosterone mediates behavioral changes caused by stress possible through increasing FKBP5 mRNA expression ${ }^{[36]}$ and altering FKBP5 methylation in the hypothalamus ${ }^{[37]}$. Animal models of stress also highlighted a role for FKBP5 in mediating coping behaviors to stress and in the mediation of stress effects $^{[38-40]}$.

Both functional and structural changes of hypothalamic have been linked to SCZ. For example, abnormalities in HPA axis has been found to play a role in the clinical manifestation of SCZ ${ }^{[41]}$. Enlarged hypothalamic volumes in schizophrenia were found SCZ patients compared to controls ${ }^{[6]}$. These findings suggest a HPA axis regulation based FKBP5 $\rightarrow$ hypothalamic $\rightarrow$ SCZ pathogenic pathway.

\subsection{FKBP5- prefrontal cortex-SCZ}

Prefrontal cortex covers the front part of the frontal lobe. This brain region has been implicated in planning complex cognitive behavior, personality expression, decision making, and moderating social behavior ${ }^{[42]}$. Both functional and/or structural abnormality of prefrontal cortex could lead to SCZ like performance in neural networks, and may account for pathological working memory in the disorder. The aberrant changes within prefrontal cortex linked to SCZ include: alterations in information processing in prefrontal corte ${ }^{[43]}$, a reduction of prefrontal cortical hubs ${ }^{[44]}$, abnormal dopamine release within the brain region ${ }^{[45,46]}$, the neural circuitry abnormality ${ }^{[47]}$, and reduced dendritic volume of prefrontal cortical neurons ${ }^{[5]}$. Moreover, many studies noted that connectivity of the prefrontal cortex is reduced in schizophrenia, particularly for intra-prefrontal cortex connectivity ${ }^{[8,9]}$.

Meanwhile, studies showed that FKBP5 is associated with both functional changes and physical alterations of prefrontal cortex. For example, decreased FKBP5 expression ${ }^{[48]}$ and disrupted GRmediated signaling pathway along with HPA axis hyperactivity resulted in neuronal atrophy in the dorsolateral (dl) - and dorsomedial (dm) - prefrontal cortex and subsequent mood-related behavior alterations ${ }^{[49]}$. Wei et al. found that exposure to chronic mild stress led to a markedly upregulated FKBP5 protein expression in the prefrontal cortex ${ }^{[50]}$. Further, deletion of FKBP51 has been suggested to play a role in preventing stress-induced prefrontal cortical synapsin loss ${ }^{[21]}$. All these studies provided evidence for the functional and structural regulation relationship between FKBP5 and prefrontal cortex, supporting a potential FKBP5 $\rightarrow$ prefrontal cortex $\rightarrow \mathrm{SCZ}$ pathogenic pathway. As a matter of fact, Basta-Kaim et al. confirmed that decreased activity of FKBP5 in the frontal cortex result in the HPA axis hyperactivity via altering $\mathrm{GR}$ function in $\mathrm{SCZ}{ }^{[51]}$.

\subsection{FKBP5- Amygdala-SCZ}

The amygdala are two almond-shaped groups of nuclei located deep and medially within the temporal lobes of the brain, the primary role of which is in the processing of memory, decision-making, and emotional reactions. Functional and structural abnormalities of amygdala have been linked to SCZ emotion related symptoms. Some studies concluded that reduced amygdala activation ${ }^{[52]}$ or implicate aberrant amygdala activity ${ }^{[53,54]}$ may underlie deficits in emotion recognition in schizophrenia, which support the involvement of amygdala activation in the positive symptoms of schizophrenia. In addition, patients suffering from SCZ present reduced volumes of the amygdala, which is partially caused by the decreases in the number of synapses ${ }^{[55]}$.

FKBP5 has been linked to emotional, memorial and learning behaviors via regulation within amygdala. For example, Attwood et al. observed that overexpressed FKBP5 in the amygdala has a direct anxiogenic effects ${ }^{[56]}$. Hadamitzky et al. showed in an animal model that, increased neuronal activities as well as overexpression of FKBP51 in the amygdala seem to trigger anxiety-like behavior in naive rats ${ }^{[57]}$. Moreover, in a drug treatment study, Sawamura et al. reported that dexamethasone treatment was associated with reduced FKBP5 mRNA expression in the amygdala after extinction learning and retention ${ }^{[58]}$. Considering the pivotal role of the amygdala in mood regulation, associative learning, and modulation of cognitive functions, alteration of FKBP5 gene expression in the amygdala may contribute differentially to these abnormalities. Integrating these discussions, a FKBP5 $\rightarrow$ Amygdala $\rightarrow$ SCZ pathogenic pathway seems reasonable.

\section{CONCLUSION}

SCZ is a complex mental health disorder, whose pathophysiology is linked to structural abnormalities and functional deficits of many brain regions. Results from this systematic review showed that, FKBP5, a protein of the immunophilin family, plays roles 
within multiple brain regions that are linked to SCZ. Dysfunction of FKBP5 could lead to functional and/ or structural changes of these brain regions, namely hypothalamus, Hippocampus, prefrontal cortex, Amygdaloid. The variance of these brain regions in turn will influence SCZ relation brain functions, including GR-responsive pathways and HPA axis activities, mood, emotion, memory and learning related behavioral alterations. Our study built up multiple potential FKBP5 $\rightarrow$ brain $\rightarrow$ SZC pathways as possible causal regulating tunnels, through which FKBP5 influence the pathogenesis of SCZ. Results from this study help to understand the mechanisms underlying FKBP5 $\rightarrow$ SCZ association.

\section{REFERENCES}

1. McGrath J, Saha S, Chant D, Welham J. Schizophrenia: a concise overview of incidence, prevalence, and mortality. Epidemiol. Rev. 2008;30:67-76.

2. McCarley RW, Wible CG, Frumin M, Hirayasu Y, Levitt JJ, Fischer IA, et al. MRI anatomy of schizophrenia. Biol Psychiatry. 1999;45:10991119.

3. Wright IC, Rabe-Hesketh S, Woodruff PW, David AS, Murray RM, Bullmore ET. Meta-analysis of regional brain volume in schizophrenia. Am J Psychiatry. 2000;157:16-25.

4. Flashman LA, Green MF. Review of cognition and brain structure in schizophrenia: profiles, longitudinal course, and effects of treatment. Psychiatr Clin North Am. 2004;27:1-18.

5. Lidow MS. Calcium signaling dysfunction in schizophrenia: a unifying approach.Brain Res Brain Res Rev. 2003;43(1):70-84.

6. Tognin S, Rambaldelli G, Perlini C, Bellani M, Marinelli V, Zoccatelli G, et al. Enlarged hypothalamic volumes in schizophrenia. Psychiatry Res. 2012;204(2-3):75-81.

7. Kalkstein S, Hurford I, Gur RC. Neurocognition in schizophrenia. curr.top. Behav. Neurosci. 2010;4:373-390.

8. Sheffield JM, Barch DM. Cognition and restingstate functional connectivity in schizophrenia. Neurosci Biobehav Rev. 2016;61:108-20.

9. Karbasforoushan H, Woodward ND. Restingstate networks in schizophrenia.Curr Top Med Chem. 2012;12(21):2404-14.

\section{ACKNOWLEDGEMENT}

This study is partly supported by Science and Technology Department of Sichuan Province (2017SZ0049, Zhe Li), and Health and Family Planning Commission of Sichuan Province (17PJ080, Zhe Li).

We would like to thank all authors for their contribution to the study.

\section{CONFLICT OF INTERESTS}

The authors claims no conflict of interests.

10. Rimol LM, Hartberg CB, Nesvåg R, FennemaNotestine C, Hagler DJ Jr, Pung CJ, et al. Cortical thickness and subcortical volumes in schizophrenia and bipolar disorder. Biol Psychiatry. 2010;68(1):41-50.

11. Binder E. B. The role of FKBP5, a cochaperone of the glucocorticoid receptor in the pathogenesis and therapy of affective and anxiety disorders. Psychoneuroendocrinology 2009;34(Suppl. 1):S186-S195.

12. Scharf SH, Liebl C, Binder EB, Schmidt MV, Muller MB. Expression and regulation of the Fkbp5 gene in the adult mouse brain. PLoS One. 2011;6:e16883.

13. Mcewen BS, Gould EA, Sakai RR. The vulnerability of the hippocampus to protective and destructive effects of glucocorticoids in relation to stress. $\mathrm{Br} \mathrm{J}$ Psychiatry Suppl. 1992;15:18-23.

14. de Kloet ER, Joels M, Holsboer F. Stress and the brain: from adaptation to disease. Nat Rev Neurosci. 2005;6:463-475.

15. Holsboer F.The corticosteroid receptor hypothesis of depression. Neuropsychopharmacology. 2000;23:477-501.

16. Lehmann ML, Brachman RA, Martinowich K, Schloesser RJ, Herkenham M. Glucocorticoids orchestrate divergent effects on mood through adult neurogenesis. J Neurosci. 2013;33:29612972.

17. Binder EB, Salyakina D, Lichtner P, Wochnik GM, Ising $M$, Pütz B, et al. Polymorphisms in FKBP5 are associated with increased 
recurrence of depressive episodes and rapid response to antidepressant treatment. Nat Genet. 2004;36(12):1319-25.

18. Binder EB, Bradley RG, Liu W, Epstein MP, Deveau TC, Mercer KB, et al. Association of FKBP5 polymorphisms and childhood abuse with risk of posttraumatic stress disorder symptoms in adults. JAMA. 2008; 299(11):1291305.

19. Yehuda R, Cai G, Golier JA, Sarapas C, Galea $\mathrm{S}$, Ising $\mathrm{M}$, et al. Gene expression patterns associated with posttraumatic stress disorder following exposure to the World Trade Center attacks. Biol Psychiatry. 2009 Oct 1;66(7):708711.

20. Mihaljevic M, Zeljic K, Soldatovic I, Andric S, Mirjanic T, Richards $A$, et al. The emerging role of the FKBP5 gene polymorphisms in vulnerability-stress model of schizophrenia: further evidence from a Serbian population. Eur Arch Psychiatry Clin Neurosci. 2016 Aug 23. PMID: 27552816

21. Schmidt U, Buell DR, Ionescu IA, Gassen NC, Holsboer F, Cox MB, et al. A role for synapsin in FKBP51 modulation of stress responsiveness: Convergent evidence from animal and human studies. Psychoneuroendocrinology. 2015;52:43-58.

22. Green MJ, Raudino A, Cairns MJ, Wu J, Tooney PA, Scott RJ, et al. Do common genotypes of FK506 binding protein 5 (FKBP5) moderate the effects of childhood maltreatment on cognition in schizophrenia and healthy controls? J Psychiatr Res. 2015;70:9-17.

23. Morimoto M, Morita N, Ozawa H, Yokoyama K, Kawata M. Distribution of glucocorticoid receptor immunoreactivity and mRNA in the rat brain: an immunohistochemical and in situ hybridization study. Neurosci Res. 1996;26:235-269.

24. Caldwell KK, Goggin SL, Tyler CR, Allan AM. Prenatal alcohol exposure is associated with altered subcellular distribution of glucocorticoid and mineralocorticoid receptors in the adolescent mouse hippocampal formation. Alcohol Clin Exp Res. 2014;38(2):392-400.

25. Fani N, Gutman D, Tone EB, Almli L, Mercer KB, Davis J, et al. FKBP5 and attention bias for threat: associations with hippocampal function and shape. JAMA Psychiatry. 2013;70:392-400.

26. Soontornniyomkij V, Risbrough VB, Young JW, Wallace CK, Soontornniyomkij B, Jeste
DV, et al. Short-term recognition memory impairment is associated with decreased expression of FK506 binding protein 51 in the aged mouse brain. Age (Dordr). 2010; 32(3):309-322.

27. Pagliaccio D, Luby JL, Bogdan R, Agrawal A, Gaffrey MS, Belden AC, et al. Stress-system genes and life stress predict cortisol levels and amygdala and hippocampal volumes in children. Neuropsychopharmacology. 2014;39(5):124553.

28. Guidotti G, Calabrese F, Anacker C, Racagni G, Pariante CM, Riva MA. Glucocorticoid receptor and FKBP5 expression is altered following exposure to chronic stress: modulation by antidepressant treatment. Neuropsychopharmacology, 2013;38(4):616-27.

29. Antoniou K, Bekris S, Saranti M, Stathis P, Rimikis M, Papadopoulou-Daifoti Z. The effects of antipsychotic drugs on serotonergic activity in the rat hippocampus.Eur Neuropsychopharmacol. 2000;10(5):315-324.

30. Grace AA. Gating of information flow within the limbic system and the pathophysiology of schizophrenia. Brain Res Brain Res Rev. 2000;31(2-3):330-341.

31. Stefanis N, Frangou S, Yakeley J, Sharma T, O'Connell P, Morgan K, et al. Hippocampal volume reduction in schizophrenia: effects of genetic risk and pregnancy and birth complications.Biol Psychiatry. 1999;46(5):697702.

32. Gothelf D, Soreni N, Nachman RP, Tyano S, Hiss $\mathrm{Y}$, Reiner $\mathrm{O}$, et al. Evidence for the involvement of the hippocampus in the pathophysiology of schizophrenia. Eur Neuropsychopharmacol. 2000;10(5):389-395.

33. Albu S, Romanowski CP, Letizia Curzi M, Jakubcakova V, Flachskamm C, Gassen NC, et al. Deficiency of FK506-binding protein (FKBP) 51 alters sleep architecture and recovery sleep responses to stress in mice. J Sleep Res. 2014;23(2):176-85.

34. Fujii T, Hori H, Ota M, Hattori K, Teraishi T, Sasayama D, et al. Effect of the common functional FKBP5 variant (rs1360780) on the hypothalamic-pituitary-adrenal axis and peripheral blood gene expression. Psychoneuroendocrinology. 2014;42:89-97.

35. White LO, Klein AM, Kirschbaum C, Kurz-Adam M, Uhr M, Müller-Myhsok B, et al. Analyzing 
pathways from childhood maltreatment to internalizing symptoms and disorders in children and adolescents (AMIS): a study protocol. BMC Psychiatry. 2015;15:126.

36. Lee RS, Tamashiro KL, Yang X, Purcell $\mathrm{RH}, \mathrm{H}$ arvey $\mathrm{A}$, Willour VL, et al. Chronic Corticosterone Exposure Increases Expression and Decreases Deoxyribonucleic Acid Methylation of Fkbp5 in Mice. Endocrinology. 2010;151:4332-4343.

37. Lee RS, Tamashiro KL, Yang X, Purcell RH, Huo $\mathrm{Y}$, Rongione $\mathrm{M}$, et al. A measure of glucocorticoid load provided by DNA methylation of Fkbp5 in mice. Psychopharmacology (Berl.) 2011;218:303-312.

38. Touma C, Gassen NC, Herrmann L, CheungFlynn J, Büll DR, lonescu IA, et al. FK506 binding protein 5 shapes stress responsiveness: modulation of neuroendocrine reactivity and coping behavior. Biol. Psychiatry 2011;70:928936.

39. Hartmann J, Wagner KV, Liebl C, Scharf SH, Wang XD, Wolf $M$, et al. The involvement of FK506-binding protein 51 (FKBP5) in the behavioral and neuroendocrine effects of chronic social defeat stress. Neuropharmacology 2012;62:332-339.

40. Guidotti G, Calabrese F, Anacker C, Racagni G, Pariante CM, Riva MA. Glucocorticoid receptor and FKBP5 expression is altered following exposure to chronic stress: modulation by antidepressant treatment. Neuropsychopharmacology. 2013;38(4):616627.

41. Walker E, Mittal V, Tessner K. Stress and the hypothalamic pituitary adrenal axis in the developmental course of schizophrenia. Annu Rev Clin Psychol. 2008;4:189-216.

42. Yang $Y$, Raine A. Prefrontal structural and functional brain imaging findings in antisocial, violent, and psychopathic individuals: a metaanalysis. Psychiatry Research. 2009;174 (2): 81-88.

43. Celada P, Puig MV, Díaz-Mataix L, Artigas F. The hallucinogen $\mathrm{DOI}$ reduces low-frequency oscillations in rat prefrontal cortex: reversal by antipsychotic drugs. Biol Psychiatry. 2008 Sep 1;64(5):392-400.

44. Gore CD, Bányai M, Gray PJ, Diwadkar V, Erdi P. Pathological effects of cortical architecture on working memory in schizophrenia. Pharmacopsychiatry. 2010 May;43 Suppl 1:S927.
45. Cutsuridis V. Neurocomputational models of brain disorders. Neural Networks. 2011;76(6):431.

46. Purkayastha S, Ford J, Kanjilal B, Diallo S, Del Rosario Inigo J, Neuwirth L, et al. Clozapine functions through the prefrontal cortex serotonin $1 \mathrm{~A}$ receptor to heighten neuronal activity via calmodulin kinase II-NMDA receptor interactions. J Neurochem. 2012 Feb;120(3):396-407.

47. Lewis DA, Hayes TL, Lund JS, Oeth KM. Dopamine and the neural circuitry of primate prefrontal cortex: implications for schizophrenia research. Neuropsychopharmacology. 1992 Feb;6(2):127-134.

48. Larrieu T, Hilal ML, Fourrier C, De SmedtPeyrusse V, Sans N, Capuron L, et al. Nutritional omega-3 modulates neuronal morphology in the prefrontal cortex along with depression-related behaviour through corticosterone secretion. Transl Psychiatry. 2014 Sep 9;4:e437.

49. Godavarthi SK, Dey P, Maheshwari M, Jana NR. Defective glucocorticoid hormone receptor signaling leads to increased stress and anxiety in a mouse model of Angelman syndrome. Hum Mol Genet. 2012;21:1824-1834.

50. Wei K, Xu Y, Zhao Z, Wu X, Du Y, Sun J, et al. Icariin alters the expression of glucocorticoid receptor, FKBP5 and SGK1 in rat brains following exposure to chronic mild stress. Int J Mol Med. 2016 Jul;38(1):337-344.

51. Basta-Kaim A, Budziszewska B, Leśkiewicz M, Fijał K, Regulska M, Kubera M, et al. Hyperactivity of the hypothalamus-pituitaryadrenal axis in lipopolysaccharide-induced neurodevelopmental model of schizophrenia in rats: effects of antipsychotic drugs. Eur J Pharmacol. 2011 Jan 15;650(2-3):586-595.

52. Aleman A, Kahn RS. Strange feelings: do amygdala abnormalities dysregulate the emotional brain in schizophrenia? Prog Neurobiol. 2005 Dec;77(5):283-298.

53. Taylor SF, Liberzon I, Tandon R, Decker LR, Koeppe RA. Symptoms associated with amygdaloid activity in schizophrenia. Biological Psychiatry. 2000;47(8):S129.

54. Andino SLG, Laine C, de Peralta RG, Gothard KL. Role of the amygdala in the control of saccadic eye movements. Clinical Neurophysiology. 2008;119(9):e166.

55. Varea E, Guirado R, Gilabert-Juan J, Martí U, Castillo-GomezE, Blasco-lbáñez JM, et al. Expression of PSA-NCAM and synaptic proteins 
in the amygdala of psychiatric disorder patients. Journal of Psychiatric Research 2012;46(2):189197.

56. Attwood BK, Bourgognon JM, Patel S, Mucha M, Schiavon E, Skrzypiec AE, et al. Neuropsin cleaves EphB2 in the amygdala to control anxiety. Nature. 2011;473:372-375.

57. Hadamitzky M, Herring A, Keyvani K, Doenlen $\mathrm{R}$, Krügel U, Bösche $\mathrm{K}$, et al. Acute systemic rapamycin induces neurobehavioral alterations in rats. Behav Brain Res. 2014 Oct 15;273:1622.

58. Sawamura T, Klengel T, Armario A, Jovanovic T, Norrholm SD, Ressler KJ, et al. Dexamethasone Treatment Leads to Enhanced Fear Extinction and Dynamic Fkbp5 Regulation in Amygdala. Neuropsychopharmacology. 2016 Feb;41(3):832-846. 